\title{
A Diesel-wind-solar-battery Micro Grid's Optimal Configuration Model Based on the Modified Non-inferior Sorting Genetic Algorithm
}

\author{
Xiupeng Chen ${ }^{1, \text { a }}$ \\ ${ }^{1}$ North China Electric Power University, Beijing, China 102206 \\ aa1124756041@qq.com
}

\begin{abstract}
Keywords: Diesel-wind-solar-battery Micro Grid; Optimal Configuration; Multi-objective Optimization; NSGA - II
\end{abstract}

\begin{abstract}
The propose of optimal configuration of micro grid is to reduce economic costs,enhance power supply reliability and improve environmental performance. This article introduces the basic structure of diesel-wind-solar-battery micro grid and power supply model. Taking the minimum annual value cost as economical efficiency's optimal object and minimum environmental management cost as environmental efficiency's optimal object, the article builds a stand-alone diesel-wind-solar-battery micro grid's optimal configuration model, in which the wind speed, temperature, illumination intensity and load level are input variables. A modified non-inferior sorting genetic algorithm is proposed to find out the solution to the type and capacity of power supply. Results show that this algorithm can comprehensively evaluate the economical and environmental efficiency under different configuration plan and provide optimization design with necessary basis.
\end{abstract}

\section{Introduction}

Micro grid is a small power distribution system composed of distributed generation, energy conversion device, load, monitoring and protection equipment, which can realize self control and management. Micro grid divides into grid-connected type and stand-alone type. Stand-alone micro grid doesn't connect with regular power grid and it meets load's need by its internal distributed generation. When there exists renewable energy sources in the micro grid, we usually need to configure energy storage system to suppress the power fluctuation. In the meantime, based on the full use of renewable energy sources, this kind of grid is more suitable to supply power for users on the islands or in the remote areas. This article will focus on the optimization design of stand-alone micro grid.

The optimal configuration of micro grid power is an important element in planning and design stage. International and domestic scholars have done a lot of research in this problem and made some achievement. Paper [1] aims at the minimization of composite costs including installation, replacement, fuel, operation and maintenance cost, and it builds mixed micro grid capacity configuration model by constraints of power shortage probability. Paper [2] focuses on diesel-wind-solar-battery and sets up power source programming model concerned with energy balance control and cost-effectiveness. It also applies genetic algorithm to solve the model. The solution algorithms above represented by genetic algorithm usually exist problems such as slow convergence, more iterative times, poor stability and so on.

This article takes the structure of stand-alone diesel-wind-solar-battery micro grid and features of power supply into full account, combining with equipment, fuel, operation and maintenance costs, and finally builds stand-alone diesel-wind-solar-battery optimal configuration model. With the optimal goal of power supply economy and environmental protection effect, the model uses modified non inferior sorting genetic algorithm ( NSGA - II) to find the optimal configuration plan of both types of power supply and its capacity. The result shows that this model can fully assess the economic and environmental effect under different configuration plan, and thus provide necessary basis for optimal design. 


\section{The Structure of Micro Grid and Power Supply Model}

\section{A. The Structure of Micro Grid}

As for the micro grid in this article, the distribution generation includes wind turbines(WT), photovoltaic arrays(PV), diesel engines(DE) and battary energy storage system(BESS $)^{[3]}$. The topology structure of a stand-alone grid is shown in Figure 1.

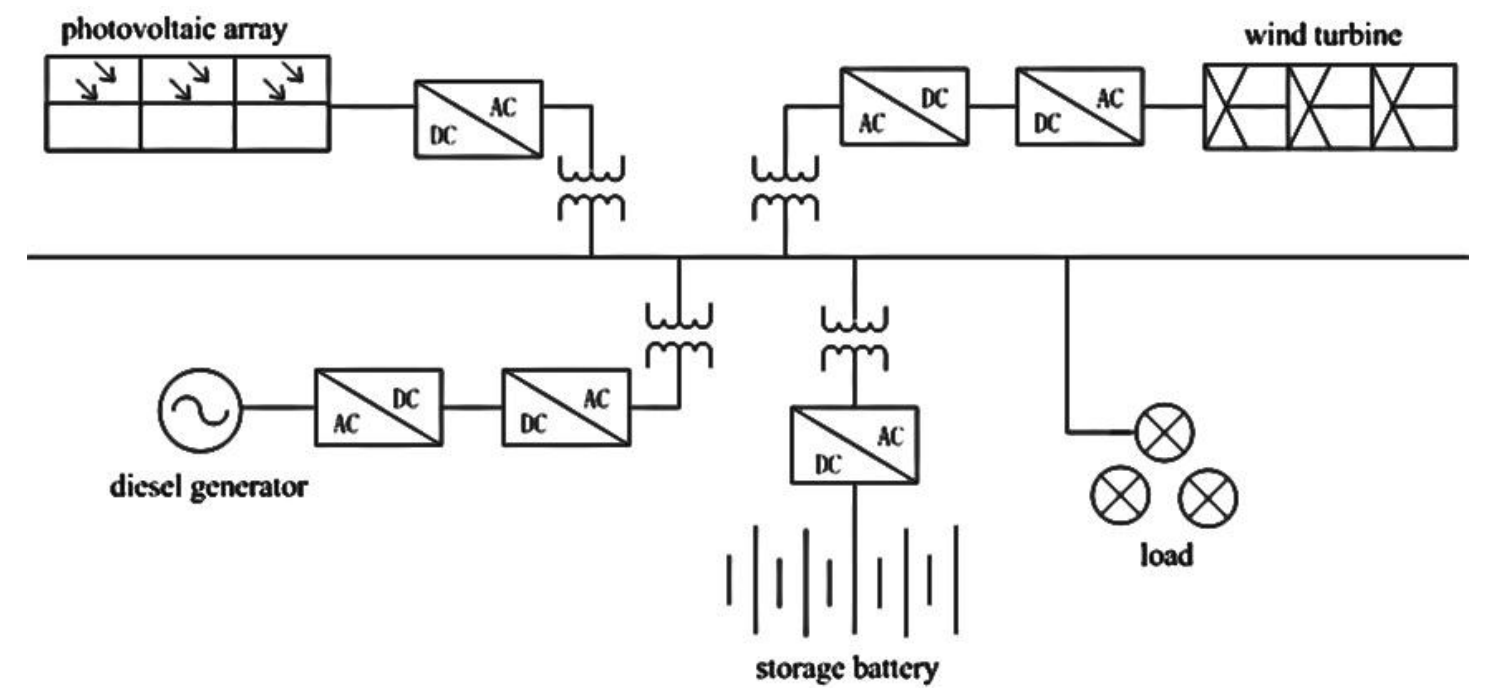

Figure 1. The structure of stand-alone micro grid

\section{B. Power Supply Model}

\section{- photovoltaic array}

Photovoltaic array is a device used to convert solar energy into electric energy. Its actual output power can be obtained through rated output power, illumination intensity and environment temperature.

$$
P_{p v-\text { out }}=P_{p s t c} \frac{G_{c}}{G_{s t c}}\left(1+k\left(T_{c}-T_{s t c}\right)\right)
$$

Where $P_{p v-o u t}$ is the actual output power of photovoltaic array, $G_{s t c}$ is the illumination intensity at standard condition $\left(1 \mathrm{~kW} / \mathrm{m}^{2}\right), G_{c}$ is the actually accepted illumination intensity, $P_{p s t c}$ is the standard output power of photovoltaic array, $k$ is power temperature coefficient, $T_{\text {stc }}$ is the surface temperature of photovoltaic array $\left(25^{\circ} \mathrm{C}\right)$, and $T_{c}$ is the battery surface temperature of operating point, which is the function of environment temperature $T_{a}$ and illumination intensity $G_{c}$.

- wind turbine

The output power $P_{w t-o u t}$ of wind turbines changes at different wind speed. The approximation relation between output power and wind speed can be expressed as the following piecewise function:

$$
P_{w t=o u t}= \begin{cases}0, & v \leq v_{c i} \\ P_{w s t c} \frac{\left(v-v_{c i}\right)}{\left(v_{r}-v_{c i}\right)}, & v_{c i}<v<v_{c o} \\ P_{w s t c}, & v \geq v_{c o}\end{cases}
$$

Where $P_{\text {wstc }}$ is the rated output power of generators, $v_{c i}$ is cut-in wind speed, $v_{c o}$ is cut-out speed and $v_{r}$ is rated wind.

- battery energy storage system 
Battery energy storage system works as energy storage element in or order to follow the change of load by controlling charge and discharge quickly. State of charge (SOC) is also an important decision variable that can avoid excessive charge-discharge. At time $t$, the state of charge of battery energy storage system is $S O C_{t}$ :

$$
S O C_{t}=\left\{\begin{array}{l}
\operatorname{SOC}_{t-1}(1-\sigma)+\frac{E_{B^{+}, t}}{E_{B}} \\
S_{O C C_{t-1}}(1-\sigma)-\frac{E_{B^{-}, t}}{E_{B}}
\end{array}\right.
$$

Where $\sigma$ is self-discharge coefficient and $E_{B}$ is the maximum capacity of battery energy storage system.

- diesel engine

Diesel engine includes diesel engine, synchronous alternator and control system. The linear relation between output power and fuel can be shown as the following formula:

$$
F=F_{0} \cdot P_{n}+F_{1} \cdot P_{\text {de-out }}
$$

Where $F, F_{0}, F_{1}$ is power consumption of diesel engines, no-load and load power consumption of generators and $P_{n}, P_{d e-o u t}$ is rated and actual output power of generators.

\section{The Scheduling Strategy of Generating Electric}

As for stand-alone micro grid with many kinds of distribution generation, the concrete control strategy for use of generation is listed as follows:

1. Put wind turbines and photovoltaic arrays into use according to the maximum generating watt in various climates.

2. When wind turbines and photovoltaic arrays can't meet the need of estrangement, battery energy storage system should be put into use on the basis of guaranteeing minimum load and discharge according to the storage calculation in the previous time.

3. If wind turbines and photovoltaic arrays can't satisfy the load's needs, diesels will start premised on the minimum generated power. During the diesels' operation, load follower mode is supposed to be adopted in order to improve the economy effect. When wind turbines or photovoltaic arrays can meet load's needs, diesels stop.

4. If the sum of generation power still can't achieve the demand, the system will cause load loss. Now the amount of load loss is an index in evaluating power distribution reliability of the system.

\section{Objective Function}

The economical efficiency of stand-alone micro grid includes initial outlay, fuel, replacement, operation and maintenance cost. Total life cycle cost can be expressed as formula below.

$$
C_{\text {total }}=C_{w t}+C_{p v}+C_{d e}+C_{\text {bess }}
$$

Where

$$
\left\{\begin{array}{l}
C_{w t}=C_{w t-i n i t}+C_{w t-o m} \\
C_{p v}=C_{p v-i n i t}+C_{p v-o m} \\
C_{d e}=C_{d e-\text { init }}+C_{d e-o m}+C_{d e-r e}+C_{f u e l} \\
C_{\text {bess }}=C_{\text {bess-init }}+C_{\text {bess-om }}+C_{\text {bess-re }}
\end{array}\right.
$$

Where $C_{\text {total }}$ is total life cycle investment cost of the system, $C_{w t}, C_{p v}, C_{d e}, C_{b e s s}$ represent investment cost of WT, PV, DE, and BESS. $C_{w t-o m}, C_{p v-o m}, C_{d e-o m}, C_{d e s s-o m}$ represent the operation 
and maintenance cost of WT, PV, DE, and DESS. $C_{w t-i n i t}, C_{p v-i n i t}, C_{d e-i n i t}, C_{d e s s-i n i t}$ represent initial outlay of them. $C_{\text {de-rep }}, C_{\text {bess-rep }}$ are the replacement cost of DE and DESS and $C_{\text {fuel }}$ is fuel cost.

Usually the lifetime of WT and PV can reach more than 20 years and whole life cycle can be treated as 20 years. As a result, in the whole life cycle, there is no replacement cost of WT and PV but there still exists replacement cost of DE and DESS. Convert the total investment cost in whole life cycle into that of annual value:

$$
C_{\text {eav }}=C_{\text {total }} \frac{i(1+i)^{l}}{(1+i)^{l}-1}
$$

$C_{\text {eav }}$ is the investment cost of annual value, $i(1+i)^{l} /\left[(1+i)^{l}-1\right]$ is capital reclamation ration, $i$ is discount rate and $l$ is project life expectation.

Take the type and amount of WT, DE, PV and DESS as decision variable and we can get the objective function as follows:

$$
\min \left(C_{\text {eav }}, E_{\text {gas }}\right)
$$

$C_{e a v}$ is objective 1 which is used to find the optimal economy efficiency of micro grid and $E_{\text {gas }}$ is objective 2 that find the optimal environment efficiency of the micro grid. When making a decision, we can change these two objectives into one objective according to the importance of different objectives. First change emissions of pollution gas into environmental management cost:

$$
C_{e n v}=\sum_{n} \alpha_{n} E_{n}
$$

Where $C_{e n v}$ is environmental management cost, $n$ is the type of pollution gas and $\alpha_{n}$ is management cost per unit of gas $n$.

After changing objective 2 into environmental management cost, we can use method of weighting to integrate with objective 1.

$$
C=\omega_{1} \cdot C_{e a v}+\omega_{2} \cdot C_{e n v}
$$

$\omega_{1}$ and $\omega_{2}$ are weight coefficients, the sum of which is 1 . For areas that have high requirement for environmental protection such as nature reserve, the $\omega_{1}$ can increase properly. While for those who lay more stress on economic results, $\omega_{2}$ will increase to some extent. In a word, weight coefficients are adjusted according to the actual conditions in order to achieve the optimal configuration.

\section{Constraint Condition}

When making optimization calculation, we should set the bounds of decision variable based on actual design requirement so that we can obtain the optimal solution within a reasonable change. In addition, stand-alone micro grid should also satisfy certain constraints.

- power balance

$$
P_{\text {load }}+P_{\text {excess }}=P_{\text {wt-out }}+P_{\text {pv-out }}+P_{\text {de-out }}+P_{\text {dess-out }}+P_{\text {unmet }}
$$

Where $P_{\text {load }}$ is load power, $P_{\text {unmet }}$ is load that can not satisfy and $P_{\text {excess }}$ is redundant load. When power supply lacks, there generates $P_{\text {unmet }}$ while if power is surplus there is $P_{\text {excess }}$. We can limit the generated power of renewable energy sources or increase load to consume power so as to achieve the power matching and stability.

- reliability

$$
\sum \frac{P_{\text {unmet }}}{P_{\text {load }}} \leq L P S P_{\text {set }}
$$

Stand-alone micro grid doesn't connect to the large grid system so the loads are all supplied by its own power supply. As a consequence, when making optimal configuration, the power distribution reliability must satisfy some constraints.

\section{- power of supply}




$$
\begin{cases}0 \leq P_{w t-o u t} \leq P_{w s t c} & \\ 0 \leq P_{p v-o u t} \leq P_{p s t c} & \text { DE off } \\ P_{d e-\text { out }}=0 & \text { DE on } \\ P_{d e-\text { min }} \leq P_{d e-o u t} \leq P_{d e-\max } & \\ P_{\text {charge-max }} \leq P_{\text {bess-out }} \leq P_{\text {incharge-max }} & \end{cases}
$$

$P_{\text {charge-max }}$ and $P_{\text {incharge-max }}$ are respectively maximum charge power and maximum discharge power.

- SOC of BESS

$$
S O C_{\min } \leq S O C_{t} \leq S O C_{\max }
$$

In the stand-alone micro grid, the time of DE's startup and shutdown is of seconds and minutes. If we calculate step in hours, DE can be treated to be able to respond to the demand change quickly without influencing the configuration results.

\section{Examples of Caculation}

This article takes a stand-alone micro grid system as research object and adopts modified non-inferior sorting genetic algorithm ( NSGA - II ) ${ }^{[4]}$ to find out the optimal configuration. Alternative power supply are WT, PV, DE and BESS. Their own model is listed in Table 1:

Table 1. Alternative types of power supply

\begin{tabular}{cc}
\hline Power supply & Alternative types \\
\hline WT & $50,100,500,780 \mathrm{~kW}$ \\
PV & Ignore the difference of types \\
DE & $250,500,1000,1500,2000 \mathrm{~kW}$ \\
BESS & Lead-acid battery $(2 \mathrm{~V} / 1000 \mathrm{Ah})$, lithium battery $(3.2 \mathrm{~V} / 180 \mathrm{Ah})$ \\
\hline
\end{tabular}

Take the wind speed in $8760 h$, illumination intensity and load as input data. $1 \mathrm{~h}$ is simulation step size and the whole time is $8760 \mathrm{~h}$. The cut-in wind speed is $2.5 \mathrm{~m} / \mathrm{s}$, cut-out wind speed is $45 \mathrm{~m} / \mathrm{s}$ and rated speed is $10 \mathrm{~m} / \mathrm{s}$. Under the premise in minimum power, diesel generators start with $30 \%$ of rated power. Assume that the density of diesel oil is $0.84 \mathrm{~g} / \mathrm{cm}^{3}$ and fuel consumption is $7.8 \mathrm{kWh} / \mathrm{L}$. Furthermore, the price of oil is $5.9 \mathrm{RMB} / \mathrm{L}$. LPSP is $1 \%$.

Since photovoltaic array, wind turbine and battery energy storage system are clean and non-polluting, their pollution emissions are ignored. But diesel engine use fuel to generate electric and produce much pollution gas. The concrete emission parameter and management cost are shown in Table 2:

Table 2. Emission and pollution treatment cost of DE

\begin{tabular}{cccc}
\hline Pollution gas & $\mathrm{NO}_{x}$ & $\mathrm{CO}_{2}$ & $\mathrm{SO}_{2}$ \\
\hline Emission $[\mathrm{g} / \mathrm{kWh}]$ & 4.33 & 232 & 0.464 \\
Pollution treatment cost[ $\mathrm{RMB} / \mathrm{kg}]$ & 62.964 & 0.21 & 14.842 \\
\hline
\end{tabular}

Here in Table 3 are the results by simulating calculation: 
Table 3. The optimal results of distributed generation

\begin{tabular}{ccc}
\hline Power supply & Type & Number \\
\hline WT & $500 k W$ & 8 \\
PV & $2410 k W$ & $/$ \\
DE1 & $250 k W$ & 2 \\
DE2 & $500 k W$ & 2 \\
BESS & lead-acid battery & 4310 \\
\hline
\end{tabular}

In this condition, $C_{\text {eav }}$ is 9.47 million yuan and $E_{\text {gas }}$ is $403900 \mathrm{~kg}$. The type of WT is selected as $500 \mathrm{~kW}$. Compared with small WT, medium and large WT possess higher cost performance. Hence, if other conditions are the same, medium and large WT have more advantages. As for BESS, types are all lead-acid batteries. Although lithium battery has longer service life and better performance, it is much more expensive. From the point of whole life cycle, all lead-acid battery has more advantages on economy than lithium battery.

Compare the results of modified non-inferior sorting genetic algorithm( NSGA - II) with those of typical quantum genetic algorithm(QGA) and common genetic algorithm(GA). The data is in Table 4:

Table 4. The comparition of the simulation results

\begin{tabular}{cccc}
\hline Algorithm & Run time[ $s$ ] & Iteration number & Optimal value[ million dollars ] \\
\hline GA & 758 & 177 & 157.36 \\
QGA & 718 & 169 & 147.34 \\
NSGA - II & 587 & 61 & 147.34 \\
\hline
\end{tabular}

As can be seen from Table 4, the algorithm presented in this article overcomes the weakness that common genetic algorithm runs into local optimum easily. In comparison to other two algorithms, NSGA - II has more advantages on rate of convergence, computational accuracy and global convergence ability.

\section{Conclusion}

This article fully takes different control strategies of stand-alone diesel-wind-solar-battery micro grid into account and build optimal configuration model based on control strategies. What's more, modified non-inferior sorting genetic algorithm is adopted to find out the solution with different economic efficiency and environmental efficiency demand. Furthermore, a concrete example shows the influence and effect that various control strategies have on optimal configuration. The results verify the rationality and validity of the model and algorithm presented in this article.

\section{References}

[1] Tafreshi S M M, Zamani H A, Ezzati S M, et al. Optimal unit sizing of distributed energy resources in microgrid using genetic algorithm[C] // Proceedings of the 18th Iranian Conference on Electrical Engineering, 2010:836-841.

[2] Jie Shu,Xianyong Zhang,Yuliang Chen,Changhong Wu.distributed micro-grid renewable energy power planning method and I ts application [J] Control Theory \& Applications,in Chinese. 2010,05: 675-680

[3] Yanhua Liu,Yafei Li,Dongmei Zhao,Guoqing contrast standalone micro-grid Power Optimization Model [J]. Modern Electric Power, in Chinese.2015,06: 14-22.

[4] Kalyanmoy Deb,Samir Agrawal,Amrit Pratap,T. Meyarivan. A fast and elitist multiobjective genetic algorithm: NSGA-II.[J]. IEEE Trans. Evolutionary Computation,2002,6. 